Research Article

\title{
Research on Bidding Game and Its Application Based on Copetition Scenario
}

\author{
Yanyong Sun \\ School of Management Harbin Institute of Technology, Harbin 150001, China \\ Correspondence should be addressed to Yanyong Sun; sunyanyong@sina.com
}

Received 12 March 2021; Accepted 5 July 2021; Published 14 July 2021

Academic Editor: Andrzej Swierniak

Copyright ( 2021 Yanyong Sun. This is an open access article distributed under the Creative Commons Attribution License, which permits unrestricted use, distribution, and reproduction in any medium, provided the original work is properly cited.

Bidding decision is not only a science, an art, but also a game. The more intense the competition, the more important the game. In practice, there is the possibility of collaboration between bidders and even hidden competing behaviors such as bidding rigging. In this study, the optimized low-price bid winning method was discussed, and the characteristics and application of the bidding game under the copetition scenarios were studied. The results show the following: (1) Under the copetition scenario, the rational bidding behavior of bidders will deviate according to the different information advantages, and there is a game of making bidding strategy decisions according to the competitive scenario. (2) There is a close functional relationship between the winning bid result and the evaluation elimination factor, the number of bidders, and the number of bidders who operate bidding rigging. (3) Based on the quotation strategy matrix modeling, it enables the quantitative decision making bid amount, offer score, and deviation risk. This study enriches the theory of quota decision in copetition scenarios and is enlightening for similar business behavior game decisions.

\section{Introduction}

Bidding is the transaction behavior of selecting qualified suppliers or builders by applying technical, economic, and market rules. When organizing the bidding for goods, projects, and services, the tenderee entrusts the tendering company to issue a bidding announcement to guide the bidders to submit the bidding documents for fair competition and the experts will examine and approve the qualified winning bidders according to the bid evaluation rules. From the practice of bidding and tendering, the bidding process has been standardized, open and transparent, but there are still many defects in the bidding system, among which the bidding rigging behavior is getting more and more hidden so that it is difficult to identify. Bidding rigging, also known as collaboration with bidding, refers to the use of unfair means between tenderers and bidders or between bidders to crowd out other bidders, thus making a stakeholder win the bid for profit. Considering the bidding rigging among bidders, bidders can be divided into two categories: bidders and independent bidders, among which bidders can be divided into main bidders and participating bidders. On the basis of the bad behavior of bidders, this paper considers three copetition scenarios, namely, independent copetition scenario, many-toone copetition scenario, and multigroup bidding scenario. In the situation of independent competition, all bidders are independent bidders, and there is no obvious interest cooperation relationship between them. In the many-to-one competition scenario, there is one bidding group and multiple (or one) independent bidders. Multigroup bidding refers to the existence of two or more bidding rigging groups, while other bidders are independent of each other.

At present, with the general improvement of bidders' qualifications and professional competence, the differences of project technology and business schemes between different bidders are becoming less and less significant, which makes the competition around price more and more fierce. According to statistics, in the evaluation of the winning bidder, the price score accounts for a large proportion, usually accounting for more than $30 \%$ of the total score. This means that the price evaluation plays an important role in deciding whether to win the bid or not, which makes the quotation strategy become the key factor affecting the winning of the bid. In accordance with the different characteristics and 
objectives of bidders, quotation strategies can generally be divided into three categories: survival strategies, competitive strategies, and profitable strategies. When bidders (enterprises) are faced with life-and-death choices, they have the characteristics of risk aversion. By adopting survival strategy, they can achieve lower bid price or higher winning probability. For the common risk neutral bidders, they generally use competitive bidding strategy to obtain reasonable profits based on the analysis of cost, quotation, and market situation. For the bidders who pursue profit maximization, they are willing to take greater risks and adopt profit-making strategy to obtain the possibility of high profit.

In the process of bidding, bidders represent different stakeholders, and they make decisions according to enterprise cost, evaluation rules, bidding purpose, etc. In practice, bidders usually cannot get comprehensive information of participants, and there is information asymmetry between them because of competition. Although the laws and regulations prohibit bidding rigging, bidding collusion, and other behaviors, the copetition behavior has the characteristics of concealment and asymmetric information. In practice, there is the possibility of cooperation between bidders, and even there are implicit competition behaviors such as bidding rigging. Based on the knowledge of decision matrix method and game theory, this paper analyzes the bidding game strategy under different copetition scenarios and realizes the quantitative analysis of bidding game, optimal bidding amount, risk of losing points, and elimination risk. In the many-to-one copetition scenarios, the bidder prefers the profit-oriented strategy to pursue more profits; however, in other copetition scenarios (i.e., independent copetition scenarios and multigroup copetition scenarios), the bidder prefer the competitive strategy, but does not choose the profitable strategy. Furthermore, the strategies of responders and competitors are similar in their rational choice order, and there is a following relationship. In addition, the result of winning the bid has a close functional relationship with the elimination coefficient of bid evaluation, the number of bidders, and the number of bidding candidates.

Section 2 reviews the research of bidding. In Section 3, the principle and related theoretical basis of the optimization method of low bid winning are introduced. Section 4 analyzes the rational quotation strategy based on the classification of quotation strategy and quotation scenario. Section 5 introduces the decision matrix to briefly describe the behavior of bidding game and analyzes the characteristics, amount, and deviation risk of bidding strategy under the multi-to-one competition and match situation. Section 6 analyzes the influence of the number of bidders on the bidding behavior in the context of competition and cooperation with a practical case. Section 7 summarizes the research conclusions and theoretical contributions of this paper and looks forward to the development direction.

\section{Literature Review}

As an international general commodity trading method, bidding can eliminate market monopoly and realize more effective allocation of resources through centralized and open competition. In the long-term theoretical research and practice, the related bidding theory is constantly improving, but also faces many problems.

First of all, when game theory was applied to the bidding process, the earliest work appeared in 1969. Au et al. used game theory and random number technology to study the project bidding problem of bidders' competition [1]. Yu pointed out that the bidding process of engineering projects is essentially a game between tenderers and bidders, and between bidders [2]. Zhang and Luo analyzed the bidding game of large-scale infrastructure projects based on the particularity of large-scale infrastructure bidding [3]. Huang studied the influence of bidding decision process and game behavior on investment decision under incomplete information [4]. He and Huang introduced the reverse auction method and constructed a multiattribute bidding game model preferred by public tenderers [5]. Li and Yuan put forward a bidding price game model considering bidders' risk attitude under the list pricing mode and studied the influence of bidding price strategy and risk attitude on their optimal price [6]. In the above bidding research based on game theory, we find that the related research is generally based on the assumption of independent bidding, which is consistent with the essence of bidding law, but different from the reality.

When considering the collusive behavior in the bidding process, Agbiboa and Besfamille pointed out that collusive behavior is the most serious and widespread in construction project bidding, and this phenomenon is common in developing and developed countries $[7,8]$. By comparing different auction forms, Milgrom found that collusion was more likely to occur in the form of public auction [9]. Padhi and Mohapatra found that the average, median, and variance of the ratio between the bid rigging bidder's quotation and the lowest price were higher than those of normal bidders and used this as a test for biding rigging [10]. From the tenderee's point of view, Zheng and Gao believe that increasing punishment and increasing the cost of collusion can effectively slow down the occurrence of collusion [11]. Tang believes that setting bidding control prices and publishing them in a timely manner can effectively control bidders' corrupt behaviors such as bidding collusion and bidding rigging [12]. From the perspective of game theory, considering the behaviors of bidding collusion and bidding rigging, this paper establishes a static game model with incomplete information among bidders and discusses the bidders with different risk attitudes.

In summary, the research on bidding quotation either does not consider the corruption in the bidding process, or does not quantitatively analyze the game behavior among bidders, or does not consider the different risk attitudes of bidders. In view of the above problems, based on the fact of behaviors like bidding rigging and bidding collusion in the bidding process, this paper quantitatively analyzes the bid strategy in different copetition scenarios from the perspective of game theory.

\section{Theoretical Basis}

3.1. Related Concepts. Incomplete information static game means that at least one participant does not know the action strategies or parameters of other parties, and 
communication barriers exist between bidders, resulting in information asymmetry [13]. The quotation of competitors has an important impact on the final result. The bidders cannot obtain the comprehensive information of the participants or need to estimate and speculate on the actions of competitors.

Risk neutrality refers to expressing the probability in different states by price, linking the states with risk preference and expected return, and uniformly pricing with risk neutrality preference. The premise of quotation is that all investors are risk neutral, or the price is determined in a risk neutral economic environment [14].

The assumption of rational person is the basic assumption of game theory; that is, all participants are rational actions [15]. Game theory studies the reasoning of people's interactive behavior, the rational choice of individuals under the interaction of interest and behavior, and the strategy of maximizing the probability of income or goal. There are two kinds of bidding games: one is the game between bidders and tenderers around the winning price, and the other is the game between bidders around the winning probability. Bidders make decisions according to enterprise cost, bid evaluation rules, bidding purpose, etc. The bidder does not know the actual bidding amount of potential bidders but can estimate the scope of project quotation.

Cost behavior, also known as cost habit, can be divided into fixed cost and variable cost. Fixed cost, also called "expense," refers to the cost that does not change with the output or sales volume of products within a certain range. Variable cost refers to the cost that the total cost changes in proportion to the change of business volume [16].

Marginal contribution reflects the value created by the project for the enterprise, which refers to the balance of sales revenue minus variable costs, also known as gross profit [17]. According to the marginal contribution analysis of cost habits, not considering the fixed cost, only consider the income formed by the variable cost of the project. If the enterprise has idle capacity, it can be accepted in principle as long as the bid price is higher than the unit variable cost.

Copetition scenario refers to the hidden copetition behavior formed by the communication and cooperation of bidders in the process of market development and business activities under the bidding rules. Furthermore, activities such as industrial cooperation, mutual supplier or service provider, alliance relationship, or strategic cooperation among bidders are also easy to form interest binding [18].

3.2. Optimized Low-Cost Bid Winning Method. Common bid evaluation methods include comprehensive evaluation method, compound pretender estimate method, and lowprice bid winning method. The traditional low price bidding method is easy to cause malicious low price competition among bidders, which leads to shoddy work and other behaviors in the process of implementation and leads to potential project quality problems, claims, additional budget, and other disputes. Therefore, an optimized low-price bid-winning method is designed, in which the elimination control line is set on the basis of the average bid price of bidders to prevent malicious low-price bid-winning, the effective lowest bid price is taken as the benchmark bid price, and the bid scores of other bidders are calculated accordingly. In addition, the bidder has specified the audited budget control price of the project in the bidding documents, and any bidding scheme higher than this price is invalid. In this paper, the optimized low-price bidding method is taken as the research object:

(1) In the tender announcement, the scores, rules, and budget control price of price review shall be specified. Set the project budget warning line, as follows:

$$
C_{\lambda Y}=Y_{B} \times \lambda,
$$

where $C_{\lambda Y}$ is budget warning line; $\lambda$ is elimination coefficient; $Y_{B}$ is budget control price.

(2) Among all bidders' quotations, the effective lowest quotation is the benchmark quotation.

In order to prevent malicious low-price competition, elimination coefficient and elimination control line are introduced on the basis of average quotation. The elimination control line is equal to the product of the average quotation of all qualified bidders and the elimination coefficient, which is recorded as

$$
\begin{aligned}
C_{A} & =\frac{1}{N} \sum_{i=1}^{N} C_{i}, \\
C_{A \lambda} & =C_{A} \times \lambda,
\end{aligned}
$$

where $i$ is the $i$ th bidder, $C_{A}$ is average quotation, and $C_{A \lambda}$ is eliminating the control line. $N$ is total number of participants in bidding.

(3) The quotation scheme below the elimination control line is invalid. Take the effective lowest quotation as the benchmark quotation, which is equal to the total score. The quotation deviation is recorded as

$$
\operatorname{Dev}_{i}=\left(\frac{C_{i}}{C_{0}}-1\right) \times 100 \%,
$$

where $C_{0}$ is effective lowest quotation, i.e., benchmark quotation, $C_{i}$ is the quotation of the $i$ th bidder, and $\operatorname{Dev}_{i}$ is deviation degree of the ith bidder's quotation.

Quotation score refers to the basic score minus the loss value of the bidder's quotation deviation, which is recorded as

$$
G_{i}=G_{0}-W \times \operatorname{Dev}_{i} \times 100 \geq 0,
$$

where $G_{i}$ is the score of the $i$ th bidder, $W$ is deviation from subweights, and $G_{0}$ is total effective lowest quotation score.

In order to focus on the bidding game research under the copetition scenario, the following assumptions are set for bidding behavior and environmental parameters:

(1) All bidders are rational bidders and have the ability to estimate the bidding environment and reason and decide the bidding strategy.

(2) Bidders can estimate the number of bidders and speculate about copetition scenarios. In the bidding 
process, stakeholders have motivation to use information advantages to bid, which leads to behaviors such as bidding rigging and bidding collusion.

(3) Bidders have the same or similar understanding of the project, and the cost behavior is almost the same. The project can make marginal contribution to the enterprise after winning the bid. The game process does not consider the influence of technology and business solutions.

To sum up, the research proposition of this paper is as follows.

Proposition 1. Under the copetition scenario, bidders' rational bidding behavior will deviate in accordance with different information advantages. Accordingly, there is a game model to make bidding strategy decision based on the copetition scenario.

Proposition 2. In the case of copetition scenario, the bid result has a close functional relationship to the elimination coefficient of bid evaluation, the number of bidders, and the number of bidding-rigging bidders.

Proposition 3. The quantitative analysis of bidding game, optimal bidding amount, and loss risk can be realized in the case of copetition scenario.

\section{Copetition Scenarios and Quotation Strategy Analysis}

Bidders mainly focus on the cost behavior of their own projects; analyze the cost composition, cost elements, and sensitive factors; and make bidding decisions. The bottom line price should consider the bidder's purchasing cost and out-of-pocket cost, etc. When the quotation is higher than the variable cost, it is regarded as marginal contribution. The bidder's quotation strategy should also analyze the enterprise's production capacity, market share, task completion rate, performance pressure, and industry competitive advantage. Therefore, the bidding game is a comprehensive decision on the operational efficiency and copetition scenarios of enterprises.

4.1. Quotation Strategy Classification. Survival strategy refers to the strategy of getting a higher probability of winning the bid with a lower price around the goal of marginal contribution. When an enterprise is faced with a life-and-death choice, as long as the price can cover the out-of-pocket cost, it can bring marginal contribution and net operating cash flow to the enterprise. First of all, bidders make decisions based on marginal contribution and out-of-pocket cost. Secondly, the bidding price must be controlled in a reasonable range that is not eliminated and not questioned. Thirdly, the influence of external factors is comprehensively analyzed such as competitors. Survival strategy belongs to risk aversion bidding behavior.
Competitive strategy refers to the bidding strategy implemented by bidders to obtain reasonable profits by analyzing information such as cost behavior, bidding characteristics, and market conditions. Firstly, it analyzes the business characteristics and quotation strategies of competitors. Secondly, it considers the enterprise's market strategy, market development ability, and market share. Finally, it sets a reasonable profit target. Competitive strategy is a risk-neutral bidding behavior.

Profit-oriented strategy refers to the bidding strategy formed by bidders around profit maximization. First of all, the enterprise is full of production capacity and tasks and has a high market share, reputation, or technical advantages. Secondly, enterprises face less pressure on task indicators. Thirdly, the cost of competitors is higher, and the probability of low-price bidding is lower. Choosing profit-oriented strategy can not only create greater profits for enterprises, but also face greater risk of quotation deviation. Profitoriented strategy belongs to risk-biased bidding behavior.

4.2. Classification of Copetition Scenarios. Concealed competition makes the bidding game more complicated. The bad behavior of bidders urges speculators to pursue interests and take unfair competition behaviors such as bidding rigging or bidding collusion. Bidding refers to the act of colluding in bidding matters, crowding out competitors or harming the interests of tenderers or other bidders by unfair means between tenderers and bidders [19]. The game behavior based on business judgment is called the bidding strategy under competition and cooperation situation.

The bidding strategy in the copetition scenarios is a game behavior on the basis of commercial judgment. According to the advantages and disadvantages of information, bidders can divide copetition scenarios into three basic types: independent bidding, many-to-one bidding, and multigroup bidding.

Independent copetition scenarios mean that all bidders are independent bidders, or there is no significant interest cooperation relationship between bidders, or they cannot get relevant business information between them. Independent copetition scenarios are static games with incomplete information. Under this condition, competitive strategy bidding is usually preferred for independent bidding to lock in reasonable profits. Second, choose the survival strategy, aiming at the marginal contribution of the project. In order to avoid the risk of losing points, the profit strategy is usually the last choice.

Many-to-one copetition scenarios, also known as single group copetition scenarios, refer to the copetition scenarios in which there exists a bidding group or interest community and one or more independent bidders. From the perspective of rational bidders, the main bidder with information advantage will participate in the bidding with upward or downward bidding behavior. First of all, the bidder will choose the profit-oriented strategy to maximize profits and prevent competitors from maliciously lowering prices. Secondly, if an enterprise has performance pressure in terms of market share, it will choose survival strategy. If the main 
bidder adopts survival strategy, the main bidder can gain absolute competitive advantage by influencing the trend of elimination control line.

Multigroup bidding refers to the copetition scenario when there are two or more bidding groups or interest communities and other parties are independent bidders. In the copetition scenario of multigroup competition, the bidding subjects must be independent; otherwise, they will form the same bidding subject. Because of the large number of bidders and the large base, each bidder has limited influence on the average quotation. Therefore, the average bid price tends to be ideal in the multigroup copetition scenarios, and the influence degree of bidding rigging is limited regardless of the upward or downward bidding rigging, and the bidder is more inclined to control the subtle trend of the average bid price to gain the bidding advantage.

4.3. Rational Quotation Strategy Analysis. Assume that all bidders are independent, rational, and risk neutral. First of all, it is relatively safe to approach the budget warning line from the perspective of risk avoidance. Under this condition, the elimination probability is zero, the probability of losing points is relatively low, and the project has profit space. Next, if the bidders are rational bidders and agree that the bidding amount approaching the budget warning line is the best, the average bidding price should be the budget warning line. At this time, the most preferred quotation is $\lambda \times C_{Y \lambda}$ and there is profit space when $\lambda \times C_{Y \lambda}>C$ is satisfied, and the project has marginal contribution when $\lambda \times C_{Y \lambda}>C_{v}$ is satisfied. Therefore, the range of rational bidding amount of independent bidders is $\left[\lambda \times C_{Y \lambda}, C_{Y \lambda}\right]$.

Bidders are divided into two categories: bidders and independent bidders. Among them, the bidder is further divided into the main bidder and the bidding participants, also known as the information superiority party. There are usually multiple or even one independent bidder, which is also called information inferior party. From the perspective of rational bidders, the main bidder has the advantage of information, and its bidding strategy is more active. Independent bidders use business information and market information to judge copetition scenarios and make coping strategies. First, analyze whether there is bidding rigging. Secondly, judge the bidding strategy of the bidder. Thirdly, put forward the coping strategies. Finally, the bidding amount is put forward according to the cost behavior and profit target of the project to prevent the risk of quotation deviation.

Under the situation of copetition scenarios, different bidders face different risks. The information superiority party has the ability to influence the average price and then can influence the quotation elimination control line. From the perspective of rational bidders, the bidder preferred the upward bidding rigging, that is, the profitable bidding strategy, in order to obtain higher project profits. At the same time, the profit-making strategy raises the average quotation and elimination control line and can eliminate the independent bidders who adopt the survival strategy. If the main bidder bids downwards, that is, adopts the survival strategy to bid at a low price, it will affect the average quotation to achieve a lower quotation and even achieve malicious low-price bid. Therefore, if the main bidder chooses the survival strategy, it can quote the low price that other independent bidders dare not follow up, so as to ensure winning the bid. That is the reason why the risk of information disadvantage is much higher than that of information advantage: if the information inferior party applies the survival strategy to quote the low price, it will face the risk of elimination by information superiority party's bidding upwards, and the risk of losing points by superiority party's bidding downwards. Therefore, the rational decisionmaking of bidders is affected by information advantage. The independent bidder should first analyze the bidding behavior in the competition and cooperation scenario, rank the strategies of the dominant party, and then put forward the corresponding strategies.

\section{Copetition Scenarios and Bidding Game Model}

5.1. Decision Matrix Model. Considering there are many strategic actions of responders and strategic states of competitors, this paper uses decision matrix method to model and analyze the bidding game and strategy under the copetition scenarios [20].

Assume there are $n$ coping strategies under copetition scenarios, which are written as the set $X=\left\{X_{1}, X_{2}\right.$, $\left.\ldots, X_{j}, \ldots, X_{n}\right\}$. It is called the response strategy action vector, and $X_{j}$ is its $j$ th component. The $m$ competitors' strategy state is written as competitor strategy state vector (set) $S=\left\{S_{1}, S_{2}, \ldots, S_{i}, \ldots, S_{m}\right\}$, where $S_{i}$ is its $i$ th component. The occurrence probability of each competitor's strategy state is written as $P=\left\{P\left(S_{1}\right), P\left(S_{2}\right), \ldots, P\left(S_{i}\right), \ldots, P\left(S_{m}\right)\right\}$. It is called the competitor strategy state probability matrix or probability matrix. According to probability theory, we obtain

$$
P\left(S_{1}\right)+P\left(S_{2}\right)+\cdots+P\left(S_{i}\right)+\cdots+P\left(S_{m}\right)=1 .
$$

The target benefit value under the competitors' strategy state $S_{i}$ and the $j$ th responder's strategy action is $x_{i j}=x\left(S_{i}, X_{j}\right)$. When $j$ is constant and $i$ changes from 1 to $m$, the profit and loss values of the coping strategy scheme $X_{j}$ under different competitor strategy states $S_{1}, S_{2}, \ldots, S_{i}, \ldots, S_{m}$ are respectively written as $x_{1 j}, x_{2 j}, \ldots, x_{i j}, \ldots, x_{n j}$. The general decision table is shown in Table 1.

According to the above analysis, the strategic plan of the responder is

$$
E\left(X_{j}\right)=x_{1 j} P\left(S_{1}\right)+x_{2 j} P\left(S_{2}\right)+\cdots+x_{i j} P\left(S_{i}\right)+\cdots+x_{m j} P\left(S_{m}\right)=\sum_{i=1}^{m} x_{i j} P\left(S_{i}\right) .
$$


TABle 1: General decision table.

\begin{tabular}{|c|c|c|c|c|c|c|}
\hline \multirow[b]{2}{*}{ Coping probability } & \multicolumn{6}{|c|}{ Responder } \\
\hline & $X_{1}$ & $X_{2}$ & $\ldots$ & $X_{j}$ & $\ldots$ & $X_{n}$ \\
\hline$P\left(S_{1}\right)$ & $x_{11}$ & $x_{12}$ & $\ldots$ & $x_{1 j}$ & $\ldots$ & $x_{1 n}$ \\
\hline$P\left(S_{1}\right)$ & $x_{21}$ & $x_{22}$ & $\ldots$ & & $\ldots$ & $x_{2 n}$ \\
\hline$\vdots$ & $\vdots$ & $\vdots$ & $\vdots$ & $\vdots$ & $\vdots$ & $\vdots$ \\
\hline$P\left(S_{i}\right)$ & $x_{i 1}$ & $x_{i 2}$ & $\cdots$ & $x_{i j}$ & $\cdots$ & $x_{i n}$ \\
\hline$\vdots$ & $\vdots$ & $\vdots$ & $\vdots$ & $\vdots$ & $\vdots$ & $\vdots$ \\
\hline$P\left(S_{m}\right)$ & $x_{m 1}$ & $x_{m 2}$ & $\ldots$ & $x_{m j}$ & $\cdots$ & $x_{m n}$ \\
\hline Expectation value of dealing with profit and loss & $E\left(X_{1}\right)$ & $E\left(X_{2}\right)$ & $\ldots$ & $E\left(X_{j}\right)$ & $\ldots$ & $E\left(X_{n}\right)$ \\
\hline
\end{tabular}

The matrix of profit and loss value $\mathbf{B}$ (also known as risk matrix) is

$$
\mathbf{B}=\left(\begin{array}{cccc}
x_{11} & x_{12} & \cdots & x_{1 n} \\
x_{21} & x_{22} & \cdots & x_{2 n} \\
\vdots & \vdots & \vdots & \vdots \\
x_{i 1} & x_{i 2} & \cdots & x_{i n} \\
\vdots & \vdots & \vdots & \vdots \\
x_{m 1} & x_{m 2} & \cdots & x_{m n}
\end{array}\right) .
$$

The expected value matrix of the coping strategy scheme is

$$
E(X)=\left(\begin{array}{llll}
E\left(X_{1}\right) & E\left(X_{2}\right) & \cdots & \left.E\left(X_{n}\right)\right)
\end{array}\right.
$$

The probability vector is written as $P=\left\{P_{1}, P_{2}, \ldots, P_{i}, \ldots, P_{m}\right\}$. Clearly, there are

$$
\begin{aligned}
& \mathbf{P} \times \mathbf{B}=\left(\begin{array}{llllll}
P_{1} & P_{2} & \cdots & P_{i} & \cdots & P_{m}
\end{array}\right)\left(\begin{array}{cccc}
x_{11} & x_{12} & \cdots & x_{1 n} \\
x_{21} & x_{22} & \cdots & x_{2 n} \\
\vdots & \vdots & \vdots & \vdots \\
x_{i 1} & x_{i 2} & \cdots & x_{i n} \\
\vdots & \vdots & \vdots & \vdots \\
x_{m 1} & x_{m 2} & \cdots & x_{m n}
\end{array}\right) \\
& =\left(\sum_{i=1}^{m} P_{i} x_{i 1} \sum_{i=1}^{m} P_{i} x_{i 2} \cdots \sum_{i=1}^{m} P_{i} x_{i j} \cdots \sum_{i=1}^{m} P_{i} x_{i n}\right) \\
& =\left(\begin{array}{llll}
E\left(X_{1}\right) & E\left(X_{2}\right) & \cdots & \left.E\left(X_{n}\right)\right)=E(X) .
\end{array}\right.
\end{aligned}
$$

So $\mathbf{P} \times \mathbf{B}=E(\mathbf{X})$. $\mathbf{B}$ has $m$ rows and $n$ columns, and matrix $\mathbf{P}$ has 1 row and $m$ columns; then, matrix $E(\mathbf{X})$ has 1 row and $n$ columns. In order to obtain the maximum target expectation in the bidding game under the copetition scenario, the solution corresponding to the largest element in the row vector of the responder's strategy plan expectation value is the optimal solution. That is,

$$
X_{\tau}=\max _{X_{j} \in A}\left\{E\left(X_{j}\right)\right\}
$$

where $X_{\tau}$ is the optimal solution of the responder strategy. If there are several coping strategies to choose from, the optimal order of decision-making is the same as the expected order.
5.2. Quote Game Using Decision Matrix. Using the decision matrix method to deal with the competitor strategy state matrix and the responder strategy action matrix, the coping strategy weight matrix is obtained as

$$
E(\mathbf{X})=\mathbf{P} \times \mathbf{B},
$$

where $\mathbf{X}$ is responder's strategy plan set \{profitable strategy, competitive strategy, and survival strategy\}; $\mathbf{P}$ is probability matrix of competitor strategy; and $\mathbf{B}$ is the profit and loss value matrix. Delphi evaluation method was introduced to quantitatively evaluate the score of $\mathbf{X}$ and $\mathbf{B}$.

5.2.1. Independent Copetition Scenarios. In the independent copetition scenarios, the competitor's profit strategy is assigned $1 / 6$, the competition strategy is assigned $1 / 2$, and the survival strategy is assigned $1 / 3$. The competitor strategy matrix $P^{\prime}$ is

$$
\left(\frac{1}{6} \frac{1}{2} \frac{1}{3}\right) \triangleq \mathbf{P}^{\prime}
$$

The responder strategy matrix $B^{\prime}$ is

$$
\left(\begin{array}{ccc}
1 & \frac{1}{2} & 0 \\
0 & 1 & 0 \\
0 & 0 & 1
\end{array}\right) \triangleq \mathbf{B}^{\prime} \text {. }
$$

Response strategy weight is

$$
E^{\prime}(\mathbf{A})=\mathbf{P}^{\prime} \times \mathbf{B}^{\prime}=\left(\frac{1}{6} \frac{7}{12} \frac{1}{3}\right) \text {. }
$$

That is, the weight of profit coping strategies is $1 / 6$, that of competition coping strategies is $7 / 12$, and that of survival coping strategies is $1 / 3$. Responders' strategies are competition, survival, and profit.

5.2.2. Many-to-One Copetition Scenario. In the situation of many-to-one copetition scenario, competitors are assigned $1 / 2$ for profit strategy, $1 / 6$ for competition strategy, and 1/3 for survival strategy. The competitor's strategy $P^{\prime \prime}$ is

$$
\left(\frac{1}{2} \frac{1}{6} \frac{1}{3}\right) \triangleq P^{\prime \prime}
$$


The responder strategy $\mathbf{B}^{\prime \prime}$ is

$$
\left(\begin{array}{lll}
1 & \frac{1}{2} & 0 \\
0 & 1 & 0 \\
0 & 0 & 1
\end{array}\right) \triangleq \mathbf{B}^{\prime \prime} .
$$

Response strategy weight $E^{\prime \prime}(\mathbf{A})$ is

$$
E^{\prime \prime}(\mathbf{A})=P^{\prime \prime} \times B^{\prime \prime}=\left(\begin{array}{lll}
\frac{1}{2} & \frac{1}{4} & \frac{1}{3}
\end{array}\right) .
$$

That is to say, the weight of profitable coping strategies is $1 / 2$, that of competitive coping strategies is $1 / 4$, and that of survival coping strategies is $1 / 3$. The responder's strategy plan selects first a profitable coping strategy, second a survival coping strategy, and last a competitive strategy.

5.2.3. Multigroup Copetition Scenarios. In the multigroup copetition scenarios, the competitor's profit-based strategy is assigned a value of $1 / 6$, the competitive strategy is assigned a value of $1 / 2$, and the survival-based strategy is assigned a value of $1 / 3$. The competitor's strategy $P^{\prime \prime \prime}$ is

$$
\left(\frac{1}{6} \frac{1}{2} \frac{1}{3}\right) \triangleq P^{\prime \prime \prime} .
$$

The responder strategy $B^{\prime \prime \prime}$ is

$$
\left(\begin{array}{lll}
1 & \frac{1}{2} & 0 \\
0 & 1 & 0 \\
0 & 0 & 1
\end{array}\right) \triangleq B^{\prime \prime \prime} .
$$

Response strategy weight is

$$
E^{\prime \prime \prime}(\mathbf{A})=P^{\prime \prime \prime} \times B^{\prime \prime \prime}=\left(\begin{array}{lll}
\frac{1}{6} & \frac{7}{12} & \frac{1}{3}
\end{array}\right) .
$$

That is to say, the weight of profitable coping strategies is $1 / 6$, that of competitive coping strategies is $7 / 12$, and that of survival coping strategies is $1 / 3$. Responders' strategies are competition, survival, and profit.

By analyzing the weight of competitors' strategies and coping strategies, we can see that the bidding game based on copetition scenarios has the following characteristics:

(1) The rational selection order of competitor strategy and the order of responder strategy are similar, and there is a following relationship between responder strategy and competitor strategy.

(2) In the scenario of many-to-one copetition, it is the most likely for the bidders with information advantage to choose the profitable strategy, and the less likely for them to choose the survival strategy.

(3) Except in the scenarios of many-to-one copetition, where the difference between the survival strategy and the profit strategy is significant, in most cases survival strategy is applicable against competitive and survival strategy.

In summary, the rational bidding behavior of bidders will deviate according to different copetition scenarios and affect the bidding game strategy. Therefore, Proposition 1 holds.

5.3. Competitive Bidding Decision under Copetition Scenarios. Static bidding game is called bidding strategy [21], which should finally be implemented to the bidding amount and deviation risk. Deviation risk includes elimination risk and loss risk. The decision-making steps of bidding amount are as follows:

(1) Determine the copetition scenarios and analyze competitors' bidding strategies by using the strategy matrix model

(2) Determine the bidding parameters, establish the quote model, and calculate the elimination control line

(3) Comprehensively analyze the bidding strategy and calculate the risk that the bidding amount will be eliminated

There are three types of bidders in the copetition scenarios: main bidder, participating bidder, and independent bidder.

Elimination control line is

$$
C_{A \lambda}=\lambda \times \frac{1}{N} \sum_{i=1}^{N} C_{i} .
$$

If there is a marker group, there are

$$
N=\sum_{i=1}^{M} N_{i} .
$$

Considering the bidding group, elimination control line is

$$
C_{A \lambda}=\lambda \times \frac{1}{N} \sum_{i=1}^{M} \sum_{j=1}^{N_{i}} C_{i j}
$$

where $M$ is the number of bidding groups; $N_{i}$ is the number of bidders in the $i$ th bidding group; $N$ is the total number of participants in bidding; $C_{i j}$ is the quotation of the bidder in the bidding group.

Assume that the party is the first bidder in the first group, and write it as $C_{11}$. Set the best quotation of the party as the elimination control line, and substitute it into (23). We obtain

$$
C_{A \lambda}=\lambda \times \frac{1}{N-1} \sum_{i=1}^{M} \sum_{j=1}^{N_{i}} C_{i j}, \quad i \times j \neq 1 .
$$

According to the analysis of strategy matrix, the information superiority of the bidder determines its bidding strategy and then influences the bidding strategy followed by 
the respondent. The elimination control line is the lower limit of the bidder's quotation, that is $C_{11} \geq C_{A \lambda}$. Considering the basic requirements of the bidder for marginal contribution, then $C_{A \lambda} \geq C_{V}$. Usually, the upper limit of the respondent's quotation is controlled in the budget warning line. Therefore, the recommended bidding amount range of respondents is

$$
C_{11} \in\left[\max \left(C_{A \lambda}, C_{V}\right), C_{Y \lambda}\right],
$$

where $C_{11}$ refers to the quotation of the respondent to be solved and $C_{V}$ is the variable cost.

Breaking through the recommended bidding amount range will face higher risk of deviation. When the bidding amount is lower than the elimination control line, it faces the risk of elimination. The farther the quotation deviating from the elimination control line, the higher the bidding amount, the greater the project income, and the higher the risk of losing points. Therefore, the bidding amount is a comprehensive decision on the expected return and bidding risk.

5.4. Strategy Characteristics in Many-to-One Copetition Scenarios. Suppose there are $N$ bids in the scenario of manyto-one competition, in which one group participates in bidding and the number of bidders is $N_{1}$. In accordance with the analysis of strategy matrix, the main bidder chooses the profitable strategy and the secondary survival strategy. Substitute it into (24), and, respectively, analyze the bid amount and characteristics of the two quotation strategies.

\subsubsection{Characteristics of Optimal Profit-Oriented Strategy.} According to the strategy matrix analysis, if the main bidder chooses the profit strategy, the profit of the project is taken as the first goal of the game, and the bidding participants are guided to bid upward. The bidding of participants tends to the budget control price, while the bidding of main bidders tends to the budget warning line. It is assumed that the rational quotation of independent bidders also tends to the budget warning line. At this point, the best quotation for the responder is

$$
C_{11}^{\prime}=\frac{\left(N-N_{1}\right) \times \lambda+N_{1}-1}{N-1} \times Y_{B}
$$

where $C_{11}^{\prime}$ is the best quotation of profit-making strategy in the situation of many-to-one competition.

If the equation consisting of $N, N_{1}, \lambda$ is defined as the scenario scaling factor, we obtain

$$
\gamma=\frac{\left(N-N_{1}\right) \times \lambda+N_{1}-1}{N-1}
$$

where $\gamma$ is the bidding coefficient of copetition scenarios.

Having $\gamma$ substituted into (26), the best quotation of the respondent is

$$
C_{11}^{\prime}=\gamma \times Y_{B}
$$

In the case of many-to-one copetition scenarios, if the bidder chooses the profit-making strategy, the best quotation of the responder can be expressed by the bidding coefficient and budget control price of the copetition scenarios. The bidding coefficient of copetition scenarios is a function related to the elimination coefficient, the number of bidders, and the number of bidding-rigging bidders and is less than 1.

When the bidder is the only independent bidder, i.e., $N-N_{1}=1$, substituting (27), the bidding coefficient of copetition scenarios is

$$
\gamma=\frac{N_{1}+\lambda-1}{N_{1}} .
$$

Equation (27) shows that $\lambda<1$; therefore, $\gamma \approx 1$. In the scenario of many-to-one competition, the number of bidders participating in bidding has a great influence on the evaluation rules and the value range of bidding amount. With the increase of the number of people participating in bidding rigging, the bidder's ability to control the winning probability is stronger, and the scenario bidding coefficient approaches 1 approximately. Under this condition, $C_{11}{ }^{\prime} \approx C_{Y \lambda}$. When the number of people participating in bidding rigging is large enough, the bidder can control the elimination control line to the early warning line tending to the budget. At this time, if the bidder does not adopt the following strategy but adopts the survival strategy to try to win the bid at a low price, the bidding amount will be lower than the budget warning line and will be eliminated. At the same time, the bidder has to accept a higher bid price.

In summary, in a competitive scenario where there is bidding rigging among bidders, the winning bid result is a closely related function of the bid evaluation elimination factor, the number of bidders, and the number of bidders who operate bidding rigging. Proposition 2 is proved.

5.4.2. Characteristics of the Optimal Survival Strategy. According to the strategy matrix analysis, if the main bidders choose survival strategy, the winning probability of the project is taken as the first goal of the game, and the bidding participants are guided to bid downward. The bidder's quotation tends to be low and controllable independently, and even quotes the extreme bidding amount that tends to zero. At this point, the quotation of independent bidders will face great risk of deviation. Substituting the extreme quotation of 0 for the bidder participating in the bidding into (24), we obtain

$$
C_{A \lambda}^{\prime}=\frac{N-N_{1}}{N-1} \times \lambda \times C_{i j}
$$

where $C_{A \lambda}^{\prime}$ is the best quotation of survival strategy in the situation of many-to-one copetition scenarios.

Under the scenario of many-to-one copetition, if the bidder chooses the survival strategy, the optimal quotation depends on the elimination coefficient, the number of bidders, the number of bidding-rigging bidders, and the bidding amount of independent bidders. When the bidder is the only independent bidder, that is, $N-N_{1}=1$, substituting it into (30), we obtain 


$$
C_{A \lambda}^{\prime}=\frac{1}{N-1} \times \lambda \times C_{i j}
$$

Therefore, the main bidder's choice of survival strategy can achieve a significant impact on the optimal quotation and control the bidding amount to the point where the independent bidder cannot follow up, even lower than the variable cost, so as to ensure winning the bid. The bidder's use of this information advantage is a malicious bidding behavior at the expense of income. This kind of bidding behavior not only breaks the expectations of other independent bidders, but also breaks the expectations of the tenderee.

\subsection{Calculation of Deviation Risk and Quotation Score.} The elimination control line is the bidding amount when the probability of winning the bid is maximum. Since there is an inverse relationship between the optimal quotation and the project profit, the bidding quotation is a comprehensive decision on the project profit and the winning probability. In practice, when the quotation is higher than the optimal quotation, the enterprise will have higher income, but also produce larger deviation degree and deviation risk.

The deviation degree of the $i$ th bidder's quotation is

$$
\operatorname{Dev}_{i}= \begin{cases}\left(C_{i} \div C_{0}-1\right) \times 100 \%, & C_{i} \geq C_{A \lambda}, \\ \text { weed out, } & C_{i}<C_{A \lambda} .\end{cases}
$$

The quotation score of the $i$ th bidder is

$$
G_{i}= \begin{cases}G_{0}-W \times \operatorname{Dev}_{i} \times 100, & C_{i} \geq C_{A \lambda} \& G_{0}>W \times \operatorname{Dev}_{i}, \\ 0, & C_{i} \geq C_{A \lambda} \& G_{0} \leq W \times \operatorname{Dev}_{i}, \\ \text { weed out, } & C_{i}<C_{A \lambda} .\end{cases}
$$

To sum up, quantitative analysis of bidding amount, risk of losing points, and risk of being eliminated are important factors of bidding strategy under the copetition scenarios. Under the copetition scenarios, the quantitative analysis of the bidding game, the optimal bidding amount, and the risk of losing points can be achieved. Therefore Proposition 3 holds.

\section{Case Analysis}

The evaluation rules and main parameters of known examples are as follows:

(1) The tenderee sets the project budget control price as 1000

(2) The effective lowest quotation is the benchmark price, which gets full marks, and $80 \%$ lower than the average effective quotation is eliminated

(3) The total score is 30 points, 1 point is deducted for $1 \%$ deviation from the benchmark price, and less than $1 \%$ is calculated by interpolation method

According to the business situation analysis, there are 8 bidders who pay the cash cost $C_{V}=400$ in the scenario of many-to-one competition. In accordance with the bidding strategy matrix, the main bidder chooses the profit-making strategy under the many-to-one copetition scenarios and guides the bidders to bid upwards.

Assume that the bid price of participants tend to budget control price $Y_{B}=1000$, the bid price of main bidders tend to budget warning line $C_{\lambda Y}=800$, and the rational bid price of independent bidders also tend to budget warning line $C_{\lambda Y}=800$. If the number of participants in bidding $N_{1}=7$, substitute it to (27), and the bidding coefficient of copetition scenarios is

$$
\begin{aligned}
\gamma & =\frac{\left(N-N_{1}\right) \times \lambda+N_{1}-1}{N-1} \\
& =\frac{(8-7) \times 80 \%+7-1}{8-1}=0.97 .
\end{aligned}
$$

Substitute the bidding coefficient of copetition scenarios into (28), and the best quotation of the respondent is $C_{11}^{\prime}=777.14$.

When the number of people participating in bidding is $N_{1}=7$, the recommended bidding amount value range is $C_{11} \in[777.14,800]$.

When $C_{11}=810$, substituting it into (3), the deviation degree of quotation is $\operatorname{Dev}_{i}=4.22 \%$.

Substituting (4), the quotation score is $G_{i}=25.77$.

Assuming that $C_{0}=C_{11}^{\prime}$, the number of bidders is from 1 to 7 . The value range and deviation risk of the bidding amount of the respondents are shown in Table 2.

It can be seen from the analysis of the data in Table 2 that with the increase of the number of bidding besiegers, the lowest quotation of the project presents a trend of change in the same direction. If the benchmark quotation deviates from the lowest quotation, the quotation deviation risk is proportional to the deviation degree. Therefore, the risk of deviation is minimum when the bidding amount is slightly higher than the lowest price. With the increase of bidding number, the bigger the copetition scenarios coefficient, the smaller the value range of the respondent's bidding amount. At this time, the main concern is the elimination risk. With the increasing number of bidding participants, the bidding coefficient of copetition scenarios approaches 1, and the elimination control line approaches the budget warning line. The smaller the copetition scenario coefficient, the larger the value range of the respondent's bidding amount. At this time, it is necessary to guard against the risk of losing points and being eliminated.

When all bidders are independent, it is also called an independent copetition scenario or an ideal copetition scenario. At this time, the copetition scenarios coefficient is equal to the elimination coefficient, and the recommended bidding amount range is $\left[\lambda \times C_{\lambda Y}, C_{\lambda Y}\right]$.

When the number of bidders is 8 , and the number of bidders participating in bidding is 7 (the blue line in Figure 1), 4 (the orange line in Figure 1), and 1 (the gray line in Figure 1), respectively, the influence on bidding strategy is shown in Figure 1. With the increase of the number of bidders, the trend line of bidding amount is significantly affected, deviating from 
TABLE 2: List of bid analysis under many-to-one scenario.

\begin{tabular}{|l|c|c|c|c|c|c|c|}
\hline Index & \multicolumn{7}{|c|}{ Parameters of the many-to-one co-petition scenarios $\left(Y_{B}=1000, \lambda=80 \%\right.}$, \\
& \multicolumn{7}{|c|}{$\left.N=8 ; G_{0}=30, W=1.\right)$} \\
\hline$N_{1}$ & 7 & 6 & 5 & 4 & 3 & 2 & 1 \\
\hline \multicolumn{7}{|c|}{ The value range of the respondent's bidding amount } \\
\hline$\gamma$ & 0.97 & 0.94 & 0.91 & 0.89 & 0.86 & 0.83 & 0.80 \\
\hline$C_{11}^{\prime}$ & 777.14 & 754.29 & 731.43 & 708.57 & 685.71 & 662.86 & 640 \\
\hline $\min \left(C_{11}\right)$ & 777.14 & 754.29 & 731.43 & 708.57 & 685.71 & 662.86 & 640 \\
\hline $\max \left(C_{11}\right)$ & 800 & 800 & 800 & 800 & 800 & 800 & 800 \\
\hline
\end{tabular}

suppose $C_{0}=C_{11}$, calculate the typical responder's quotation score and elimination risk.

\begin{tabular}{|c|c|c|c|c|c|c|c|}
\hline 600 & $x$ & $x$ & $x$ & $x$ & $x$ & $x$ & $x$ \\
\hline 625 & $x$ & $\times$ & $\times$ & $x$ & $\times$ & $\times$ & $x$ \\
\hline 640 & $x$ & $\times$ & $\times$ & $x$ & $x$ & $\times$ & 30 \\
\hline 650 & $x$ & $\times$ & $x$ & $x$ & $\times$ & $\times$ & 28.44 \\
\hline 662.86 & $x$ & $x$ & $x$ & $x$ & $x$ & 30 & 26.43 \\
\hline 675 & $x$ & $x$ & $x$ & $x$ & $\times$ & 28.17 & 24.53 \\
\hline 685.72 & $x$ & $x$ & $\times$ & $x$ & 30 & 26.55 & 22.86 \\
\hline 700 & $x$ & $x$ & $x$ & $x$ & 27.92 & 24.40 & 20.63 \\
\hline 708.58 & $x$ & $x$ & $\times$ & 30 & 26.67 & 23.10 & 19.28 \\
\hline 725 & $x$ & $\times$ & $x$ & 27.68 & 24.27 & 20.63 & 16.72 \\
\hline 731.43 & $x$ & $\times$ & 30 & 26.77 & 23.33 & 19.65 & 15.71 \\
\hline 750 & $x$ & $\times$ & 27.46 & 24.15 & 20.63 & 16.85 & 12.81 \\
\hline 754.29 & $x$ & 30 & 26.87 & 23.55 & 20.00 & 16.21 & 12.14 \\
\hline 775 & $x$ & 27.25 & 24.04 & 20.63 & 16.98 & 13.08 & 8.91 \\
\hline 777.15 & 30 & 26.97 & 23.75 & 20.32 & 16.67 & 12.76 & 8.57 \\
\hline 800 & 27.06 & 23.94 & 20.63 & 17.10 & 13.33 & 9.31 & 5.00 \\
\hline 825 & 23.84 & 20.63 & 17.21 & 13.57 & 9.69 & 5.54 & 1.09 \\
\hline 850 & 20.63 & 17.31 & 13.79 & 10.04 & 6.04 & 1.77 & 0 \\
\hline 875 & 17.41 & 14.00 & 10.37 & 6.51 & 2.40 & 0 & 0 \\
\hline 900 & 14.19 & 10.68 & 6.95 & 2.98 & 0 & 0 & 0 \\
\hline 925 & 10.97 & 7.37 & 3.54 & 0 & 0 & 0 & 0 \\
\hline 950 & 7.76 & 4.05 & 0.12 & 0 & 0 & 0 & 0 \\
\hline 975 & 4.54 & 0.74 & 0 & 0 & 0 & 0 & 0 \\
\hline 1000 & 1.32 & 0 & 0 & 0 & 0 & 0 & 0 \\
\hline
\end{tabular}




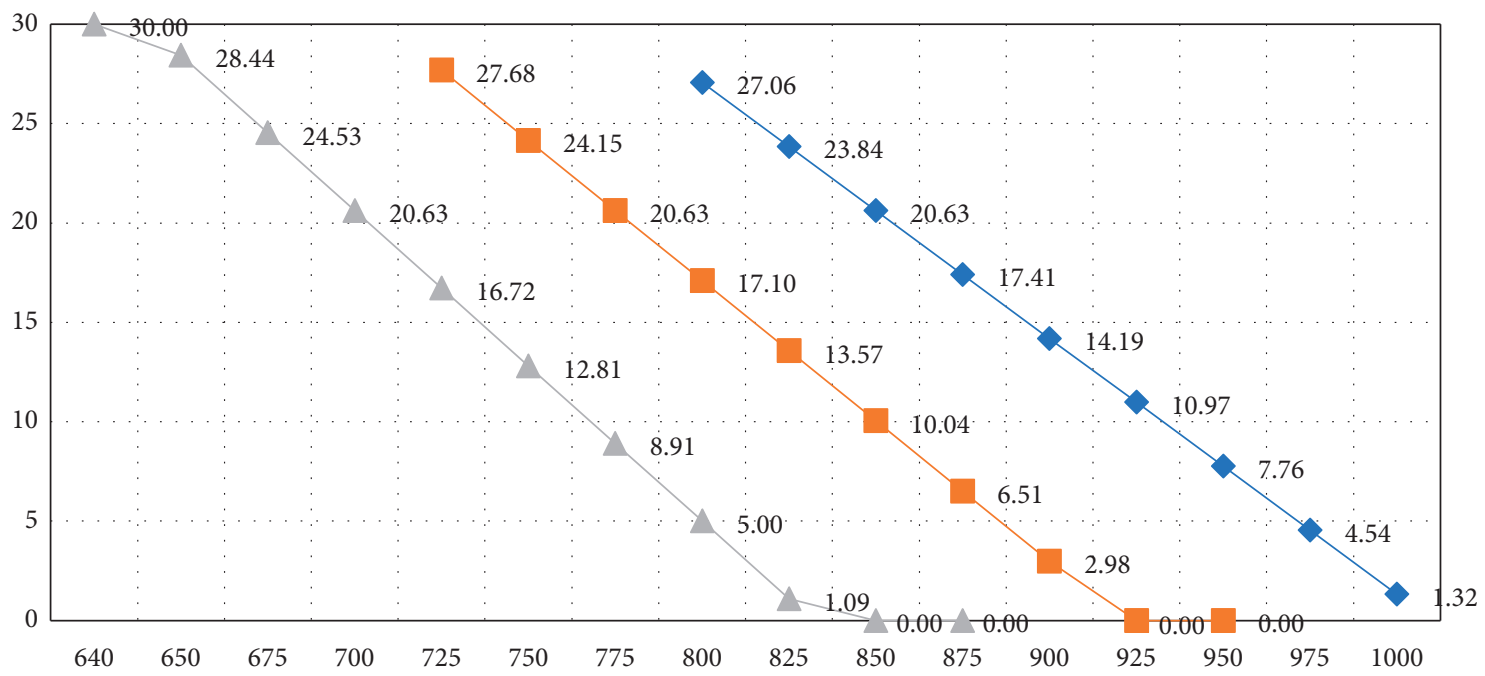

FIGURE 1: The influence trend of the number of bidders on the bidding strategy under the many-to-one copetition scenarios.

the trend line of bidding amount of rational independent bidders to the right. At this time, the tenderee bears higher bid price, and the independent bidder faces greater uncertainty and deviation risk of bid amount, and the value range of bid amount of the bidder is overall improved. The bidding rigging of the main bidder is positively related to the income, and the risk of quotation deviation of other bidders is further amplified by the game advantage.

To sum up, when judging the copetition scenarios and analyzing the bidding game, using the following strategy can effectively eliminate the asymmetric information caused by bidding behavior.

\section{Conclusions}

Bidding quotation is a complex decision full of uncertainty; a systematic project that relies on factors such as experience, intuition, and preference; and a comprehensive decision on cost level, motivation to win, and evaluation methods. In this study, the optimized low-price bidding method was taken as the research object, and the bidding strategy and scenario were modeled and analyzed theoretically by using the decision matrix tool, and the theoretical research on the influence of bidding rigging behavior on bid evaluation rules was carried out. The results of the study show that, first, the rational bidding behavior of bidders in the copetition scenario deviates depending on the information advantage, and there exists a game model for making bidding strategy decisions based on the competitive scenario. Secondly, under the copetition scenarios, there is a close functional relationship between the winning result and the elimination coefficient of bid evaluation, the number of bidders, and the number of bidders who operate bidding rigging. Thirdly, based on the modeling of quotation strategy matrix, the quantitative decision of bidding amount, quotation score, and deviation risk is realized. Finally, through the case analysis of the best quotation under the many-to-one copetition scenario, the bidding coefficient under the competition scenario was introduced, and it was demonstrated that the number of bidders participating in the bidding has a significant impact on the evaluation rules and the bidding amount range. The research results of this paper enrich the game theory of business behavior decisions under copetition scenarios and are enlightening for similar business behaviors. Due to the diversity of bid evaluation rules and the online system application of the evaluation process, information will become increasingly asymmetric between different stakeholders. Therefore, there is still a gap in the research on the precision of the bidding game strategy under the copetition scenario, and it is impossible to give consideration to both the winning probability and the project income. However, with the improvement of social informationization, it is the future research direction to improve the decision-making model by using business big data and to promote the collection of game parameters through industry data, model, and bidding behavior and environmental factors evaluation, so as to reduce the impact of illegal bidding behaviors such as bidding rigging and bidding collusion.

\section{Data Availability}

The data used to support the findings of this study are available from the corresponding author upon request.

\section{Conflicts of Interest}

The author declares no conflicts of interest.

\section{Acknowledgments}

This research was supported by the National Natural Science Foundation of China (71729001). 


\section{References}

[1] T. Au, R. L. Bostleman, and E. W. Parti, "Construction management game-deterministic model," Journal of the Construction Division, vol. 95, no. 1, pp. 25-38, 1969.

[2] $\mathrm{H}$. Yu, The General Theory of Bid Invitation and Bidding, China Geological Publishing House, Beijing, China, 1990.

[3] J. Zhang and H. B. Luo, "Game theory analysis of bidding tactics of large-scale infrastructure project," Construction Management Modernization, vol. 4, pp. 28-31, 2005.

[4] Z. X. Huang, "Modeling bidding decision in engineering field with incomplete information: a static game-based approach," Advances in Mechanical Engineering, vol. 8, no. 1, pp. 1-8, 2016.

[5] J. H. He and Y. Huang, "Research on multi-attribute bidding game of government projects when tenderer's preference is public," Systems Engineering, vol. 34, no. 2, pp. 95-102, 2016.

[6] D. F. Li and L. Yuan, "Game-based bidding quotation model considering attitude under the bill of quantities," Control and Decision, vol. 33, no. 6, pp. 1093-1099, 2018.

[7] D. E. Agbiboa, "Between corruption and development: the political economy of state robbery in Nigeria," Journal of Business Ethics, vol. 108, no. 3, pp. 325-345, 2013.

[8] M. Besfamille, "Collusion in local public works," International Economic Review, vol. 45, no. 4, pp. 1193-1219, 2004.

[9] P. Milgrom, "Putting auction theory to work: the simultaneous ascending auction," Journal of Political Economy, vol. 108, no. 2, pp. 245-272, 1998.

[10] S. S. Padhi and P. K. J. Mohapatra, "Detection of collusion in government procurement auctions," Journal of Purchasing and Supply Management, vol. 17, no. 4, pp. 207-221, 2011.

[11] C. X. Zheng and X. Gao, "Analysis on the collusion tender problem in the construction bidding process," Journal of Engineering Management, vol. 23, no. 3, pp. 229-231, 2009.

[12] Y. Tang, "Consideration on setting up bidding control price for engineering construction projects," Cities and Towns Construction in Guangxi, vol. 5, pp. 123-125, 2009.

[13] W. Y. Zhang, Game Theory and Information Economics, Joint Publishing, Shanghai, China, 1996.

[14] X. Z. Zhang and Z. Z. Zou, "Bidding model under incomplete information," Value Engineering, vol. 16, pp. 8-9, 2017.

[15] G. Q. Huang and X. He, "Non bottom tender and offering game theory model study of project bidding," Shan Xi Architecture, vol. 34, no. 9, pp. 29-30, 2008.

[16] Accounting Qualification Evaluation Center of the Ministry of Finance, Advanced Accounting Practice, Economic Science Press, Beijing, China, 2019.

[17] J. T. Zhang, Y. M. Yue, and Y. H. Yang, "Intergraded optimization system of bidding decision making based on unit price contract," Value Engineering, vol. 18, pp. 5-7, 2018.

[18] Y. Huang, X. L. Zhang, and H. Y. Wang, "Analysis of the behavior of collusive tendering in the project tender and bidding market based on game theory," Transactions of Shen Yang Li Gong University, vol. 26, no. 6, pp. 23-27, 2007.

[19] H. Y. Chen, "Analysis of bidding strategy under bill of quantities pricing mode," Value Engineering, vol. 16, pp. 15-16, 2017.

[20] Y. J. Ou, Decision Management Theory, Method, Skill and Application, Zhongshan University Press, Guangzhou, China, 2003.

[21] Q. J. Chen and B. D. Liang, "Study of game-based bidding quotation model under the bill of quantities," Science ¿Technology Progress and Policy, vol. 29, no. 18, pp. 62-65, 2012. 